\title{
Mini-Percoll Technique Induces Similar Capacitation Features in Domestic Ruminant Frozen-Thawed Spermatozoa Regardless of the Presence of Heparin
}

\author{
Vivian Angélico Pereira Alfradique ${ }^{1}$, Carolina Cerqueira Sarmento Olivares ${ }^{1}$, \\ Joanna Maria Gonçalves de Souza-Fabjan', Jeferson Ferreira da Fonseca ${ }^{2}$, Ribrio Ivan Tavares Pereira Batista', \\ Mário Felipe Alvarez Balaro', Helena Fabiana Reis de Almeida Saraiva', \\ Luana Rangel Côrtes' ${ }^{1}$ \& Felipe Zandonadi Brandão'
}

\begin{abstract}
Background: Sperm capacitation is a process consists of a series of functional, biochemical, and biophysical modifications that render the ejaculated sperm competent for oocyte fertilization. Secreted by the female reproductive tract epithelium, heparin promotes capacitation by binding to and removing seminal plasma proteins, which are adsorbed to the sperm PM and would inhibit capacitation. There is substantial evidence that cryopreservation promotes capacitation-like changes in bull, ram and buck sperm. Our general hypotheses were: (a) cryopreserved ram sperm suffer capacitation more quickly than buck and bull sperm under the same conditions; (b) the capacitation status of ruminant cryopreserved sperm is similar whether or not heparin is present after the mini-Percoll technique; and (c) ruminant frozen-thawed sperm selected by miniPercoll and incubated within media without heparin supplementation is not impaired in terms of capacitation status and sperm agglutination. This study aimed to compare sperm parameters of ovine, caprine, and bovine frozen-thawed sperm after mini-Percoll processing followed by incubation with or without heparin supplementation.

Materials, Methods \& Results: Commercial semen of all species were used. Sperm samples were selected by mini-Percoll and supplemented (or not) with heparin within an incubation medium for $18 \mathrm{~h}$. Sperm kinematics (CASA system analyzes), capacitation status (CTC staining) and sperm agglutination were evaluated after thawing, mini-Percoll, $1.5 \mathrm{~h}, 3 \mathrm{~h}, 6 \mathrm{~h}$ and $18 \mathrm{~h}$. In comparison with post-thawing analysis, ovine species demonstrated a reduction $(P<0.05)$ in most of the sperm motility parameters after mini-Percoll. Conversely, ovine samples presented the highest $(P<0.05)$ rate of acrosome-reacted cells after mini-Percoll. Heparin supplementation did not affect most of the parameters evaluated $(P>0.05)$. In caprine and bovine species, a lower $(P<0.05)$ rate of sperm agglutination was observed in the presence of heparin at $18 \mathrm{~h}$ of incubation. In the absence of heparin, ovine samples showed a higher $(P<0.05)$ agglutination rate compared to the bovine species after long incubation period.

Discussion: The present study compared sperm parameters (sperm kinematics, agglutination rate and capacitation status) of ruminant frozen-thawed sperm after mini-Percoll selection followed by in vitro incubation with or without heparin supplementation. In this study, it was observed the same rate of capacitated cells after the sperm selection (min-Percoll) between ruminant species. This indicate that the capacitation process occurs similarly between ruminant species, refuting the first hypothesis of this study. The presence of heparin did not influence the capacitation status of ruminant frozen-thawed sperm after mini-Percoll selection, it demonstrates that the second hypothesis was supported by this study making more economic and practical the use of ruminant frozen-thawed semen. The absence of heparin in the incubation medium did not harmed the capacitation status and sperm agglutination of ruminant frozen-thawed sperm. This supported the third hypothesis of the current study and indicate that the use of mini-Percoll technique regardless the presence of heparin could be a useful alternative for the preparation of ruminant frozen-thawed sperm. In conclusion, the capacitation status of ruminant frozenthawed sperm is similar whether or not heparin is present after the mini-Percoll technique.
\end{abstract}

Keywords: bovine, caprine, CTC, ovine, sperm capacitation. 


\section{INTRODUCTION}

Sperm capacitation in mammals is a prerequisite for successful fertilization [2]. It is well documented in the literature that glycosaminoglycans such as heparin play a crucial role in the capacitation process in vitro [26,18]. In ruminants, such as ovine, caprine, and bovine species, heparin also produces an increase of sperm intracellular $\mathrm{pH}, \mathrm{Ca}^{2+}$ concentration, protein phosphorylation, and modification of motility parameters [4].

Reproductive biotechnologies such as semen cryopreservation and sperm processing with the Percoll gradient, routinely used in in vitro embryo production laboratory for in vitro fertilization, induce modifications in the sperm PM that trigger premature capacitation. Although sperm cryocapacitation occurs in all ruminant species, ram sperm have a very low cholesterol/phospholipid ratio and are more sensitive to cooling and cold-shock stress than the sperm of other species [22]. Thus, there is a lot of effort to minimize the detrimental effects of cryopreservation and thawing on the ram sperm membrane [27]. To our knowledge, there are no studies comparing the capacitation status of frozen-thawed sperm between ram and other ruminant species. Therefore, this study aimed to compare sperm motility parameters, agglutination rate, and capacitation status of ovine, caprine, and bovine frozen-thawed sperm after mini-Percoll selection followed by in vitro incubation with or without heparin supplementation.

\section{MATERIALS AND METHODS}

All the experiments were carried out at Fluminense Federal University (UFF), Niteroi (Brazil, latitude $22^{\circ} 53^{\prime} \mathrm{S}$, longitude $43^{\circ} 06^{\prime} \mathrm{W}$ ). The procedures were approved by the local ethics committee (protocol approval: 374/13).

\section{Reagents}

All chemicals were purchased from Sigma Chemicals ${ }^{1}$.

\section{Experimental design}

To evaluate sperm motility and capacitation parameters in caprine $(n=3$; Alpine), ovine $(n=3$; Santa Inês), and bovine ( $\mathrm{n}=3$; Holstein) species, commercial frozen-thawed semen of nine animals of proven fertility were used. Semen $0.25 \mathrm{~mL}$ straws from each animal were maintained in liquid $\mathrm{N}_{2}$ until analy- sis. Six replicates were performed. For each replicate, two straws of each animal per species (i.e., six straws for each species) were thawed in a water bath at $37^{\circ} \mathrm{C}$ for $30 \mathrm{~s}$. This pool was placed into a $15 \mathrm{~mL}$ conical centrifuge tube (Corning Incorporated) ${ }^{2}$, from which a sample was obtained (after thawing) to determine motility and capacitation status. The remainder of the pool was divided into five aliquots of $0.15 \mathrm{~mL}$ each and submitted to sperm selection with the mini-Percoll gradient (section 2.3). After this procedure, an aliquot of each pool was used to evaluate motility rate and capacitation status. Samples of each remaining aliquot ( $n=4$ for each species) were divided into two parts for incubation in the SP-TALP medium $[100 \mathrm{mM} \mathrm{NaCl}$, $3.1 \mathrm{mM} \mathrm{KCl}, 25 \mathrm{mM} \mathrm{NaHCO}, 0.3 \mathrm{NaH}_{2} \mathrm{PO}_{4}, 21.6$ $\mathrm{mM}$ lactate (sodium salt), $2.0 \mathrm{mM} \mathrm{CaCl}_{2}$, and $10 \mathrm{mM}$ Hepes] supplemented with $6 \mathrm{mg} / \mathrm{mL}$ bovine serum albumin fraction $\mathrm{V}, 2.2 \mathrm{mg} / \mathrm{mL}$ sodium pyruvate, 50,000 $\mathrm{IU} / \mathrm{mL}$ penicillin, and $50 \mathrm{mg} / \mathrm{mL}$ streptomycin [17]. In the Heparin group, the SP-TALP medium was supplemented with $5 \mu \mathrm{g} / \mathrm{mL}$ of heparin $[8,25]$; the Control group received SP-TALP medium without heparin. Incubation occurred at $38.5^{\circ} \mathrm{C}$ under humidified atmosphere with $5 \% \mathrm{CO}_{2}$ for $18 \mathrm{~h}$. Samples from each species and treatment were obtained at intervals (1.5 h, $3 \mathrm{~h}, 6 \mathrm{~h}$, and $18 \mathrm{~h}$ ) during incubation, and analysis of agglutination rate, motility, and capacitation status was performed.

\section{Mini-Percoll technique}

The sperm was selected by the mini-Percoll technique in accordance with the method we previously established in sheep [15]. The gradient was formed by pipetting $0.4 \mathrm{~mL}$ of $90 \%$ Percoll solution into a $1.5 \mathrm{~mL}$ microtube and then overlaying it with $0.4 \mathrm{~mL}$ of $45 \%$ Percoll solution. A $0.15 \mathrm{~mL}$ aliquot of semen was placed on top of the $45 \%$ layer and then subjected to a $5000 \mathrm{x} g$ centrifugation for $5 \mathrm{~min}$. After the centrifugation, the supernatant was removed, and the pellet was resuspended in $0.4 \mathrm{~mL}$ of SP-TALP. Then, another centrifugation was performed with the same force and time. After the second centrifugation, the supernatant was discarded, and the pellet was resuspended in $0.25 \mathrm{~mL}$ of SP-TALP.

\section{Incubation media}

Immediately post-selection, the content of the mini-Percoll microtubes (4 per species) was transferred to a $15 \mathrm{~mL}$ conical centrifugal tube and leveled to a volume of $1 \mathrm{~mL}$ with SP-TALP. The total content 
was divided into either (a) SP-TALP medium without heparin (Control group) or (b) SP-TALP with $5 \mu \mathrm{g} / \mathrm{mL}$ heparin (Heparin group). The treatments comprised a total content of $1 \mathrm{~mL}$, which was divided into four 0.25 $\mathrm{mL}$ aliquots. These aliquots were transferred into four microtubes, and the samples were completed with 0.5 $\mathrm{mL}$ SP-TALP. The microtubes were then incubated, and samples were assessed at different incubation intervals.

\section{Sperm capacitation status}

The capacitation status was assessed by the chlortetracycline (CTC) test as described by [16]. A $0.75 \mathrm{mM}$ CTC solution was prepared daily in a buffer containing $20 \mathrm{mM}$ Tris, $130 \mathrm{mM} \mathrm{NaCl}$, and $5 \mathrm{mM}$ cysteine, $\mathrm{pH}$ 7.8. For staining, $0.01 \mathrm{~mL}$ of the sperm sample was mixed with $0.01 \mathrm{~mL}$ of CTC solution on a glass slide. Finally, a drop of an antifade solution (0.22 M 1,4-diazabicyclo [2.2.2] octane, DABCO $)^{1}$ was added to slow the fading of CTC fluorescence. For evaluation of CTC patterns, the samples were observed under a microscope (Nikon Eclipse Ci) ${ }^{3}$ under epifluorescence illumination at 1000x magnification with oil immersion. Two hundred sperm per slide were scored [4] and classified into three groups, as described by [5]: bright fluorescence over the whole head (noncapacitated cells, F pattern), fluorescence-free band in the post-acrosomal region (capacitated cells, B pattern), and full fluorescence over the whole head except for a thin, bright band of fluorescence along the equatorial region (acrosome-reacted cells, AR pattern).

Particularly for the CTC assay at post-thawing (PT), sperm washing was necessary to remove the semen extender particles that otherwise bind to CTC and hinder the evaluation. Therefore, sperm washing procedure was done prior to all CTC assay at PT. For this, $4 \mathrm{~mL}$ of phosphate-buffered saline was layered on top of semen within a $15 \mathrm{~mL}$ conical centrifuge tube. The tube was centrifuged twice at $200 \times \mathrm{g}$ for $6 \mathrm{~min}$. After aspiration of the supernatant, the sperm were resuspended in $500 \mu \mathrm{L}$ of phosphate-buffered saline. Finally, the washed sperm were subjected to the CTC staining assay, as described above.

\section{Sperm motility}

Sperm motility parameters were analyzed by computer-assisted semen analysis (CASA) using the SCA ${ }^{\circledR}$ system (Sperm Class Analyzer) ${ }^{4}$ connected to a microscope. The following settings were applied for all species studied: images were collected at a speed of 25 images per second, and the microscope was set to $\mathrm{x} 100$ magnifying power. Aliquots of $10 \mu \mathrm{L}$ were placed on prewarmed slide $\left(37^{\circ} \mathrm{C}\right)$ and covered with a $24 \times 24 \mathrm{~mm}$ coverslip. Afterward, measurements were performed is surplus to requirements. Sperm movement was observed under negative phase contrast optics and video-recorded in three to five different fields, with at least 500 sperm captured for analysis. Software settings were adjusted to the sperm of each evaluated species. The main software settings were as follows: The sperm head dimensions detected by the system were $18-60 \mu \mathrm{m}^{2}$ for ovine and caprine sperm, and $25-60 \mu \mathrm{m}^{2}$ for bovine sperm. Sperm were identified as immotile if the curvilinear velocity (VCL) was $<10 \mu \mathrm{m} / \mathrm{s}$, slow if $10-45 \mu \mathrm{m} / \mathrm{s}$, medium if $45-75 \mu \mathrm{m} / \mathrm{s}$, and rapid if $>75 \mu \mathrm{m} / \mathrm{s}$. The sperm cells presenting straightness (STR) $>80 \%$ were described as progressive. The following motility patterns were analyzed: total motility (\%), progressive motility (\%), average path velocity (VAP; $\mu \mathrm{m} / \mathrm{s})$, VCL $(\mu \mathrm{m} / \mathrm{s})$, straight line velocity (VSL; $\mu \mathrm{m} / \mathrm{s}$ ), straightness (STR: VSL/VAP; \%), linearity (LIN: VSL/VCL; \%), wobble (WOB: VAP/VCL; \%), lateral head displacement (ALH; $\mathrm{mm})$, and beat cross frequency $(\mathrm{BCF} ; \mathrm{Hz})$.

\section{Agglutination rate}

The evaluation of agglutination rate was performed during the motility assessment. Approximately 250 motile sperm cells were visually examined in four different fields. The percentage of head-to-head agglutinated sperm was defined as the number of sperm agglutinated to at least one other sperm per total motile sperm [28].

\section{Statistical analysis}

Statistical analysis was performed using Prism 5.01 software $^{5}$. Results are presented as mean and standard error of the mean (SEM). All quantitative variables were subjected to normality (Lilliefors test) and homoscedasticity (Bartlett test) tests. One-way analysis of variance (ANOVA) with repeated measures was performed at all different time intervals during incubation and when ANOVA revealed a significant effect, values were compared by Tukey's post hoc test. The non-normal variables were submitted to Friedman test followed by Dunn's test. Paired T test or Wilcoxon test (non-normal variables) was performed to compare different treatments (heparin group vs control group) and moments of evaluations (after thawing group vs after mini-Percoll). When $P$ value was $<0.05$, the means were considered to differ significantly. 


\section{RESULTS}

Ovine, caprine and bovine sperm motility and capacitation status before and after mini-Percoll processing

Sperm motility parameters of the three species after thawing and mini-Percoll processing are presented in Table 1. After mini-Percoll selection, a reduction $(P<0.05)$ in most of the sperm motility parameters was observed in ovine species.

The total motility and progressive motility after mini-Percoll selection were higher $(P<0.05)$ in caprine sperm compared to ovine sperm. A lower $(P<0.05)$ percentage of capacitated cells was observed in the ovine species compared with bovine species after thawing and after mini-Percoll selection. In contrast, ovine species presented the highest $(P<0.05)$ rate of acrosome-reacted cells after processing with mini-Percoll.

Ovine, caprine and bovine sperm motility and capacitation status after incubation in the presence or absence of heparin

Regardless of the species and the moment of evaluation $(1.5,3,6$, or $18 \mathrm{~h})$, heparin supplementation in the sperm incubation medium resulted in no extra benefits $(P>0.05)$ for the most parameters (except STR and WOB in ovine specie) associated with sperm motility (data are not shown). Similarly, heparin did not affect $(P>0.05)$ the rates of capacitation or acrosome-reacted (except at $18 \mathrm{~h}$ in caprine species) cells in comparison to control group. Regardless the presence of heparin, a higher $(P<$ 0.05 ) proportion of capacitated cells was observed in the ovine species compared to bovine (absence of heparin) and caprine species (presence of heparin). Conversely, a lower $(P<0.05)$ percentage of acrosome-reacted cells was noticed in the ovine species compared to bovine (absence of heparin) and caprine species (presence of heparin).

There was a species effect $(P<0.05)$ on the rate of acrosome-reacted cells; heparin supplementation resulted in higher values at $18 \mathrm{~h}$ in caprine species (Table 2).

Ovine, caprine and bovine sperm agglutination rate after incubation in the presence or absence of heparin

At $18 \mathrm{~h}$, a lower $(P<0.05)$ rate of caprine and bovine sperm agglutination was observed in the group supplemented with heparin compared to the control group (Figure 1). Additionally, in the absence or presence of heparin, ovine sperm presented higher $(P<$ $0.05)$ agglutination compared to the bovine species after long incubation periods (6-18 h).

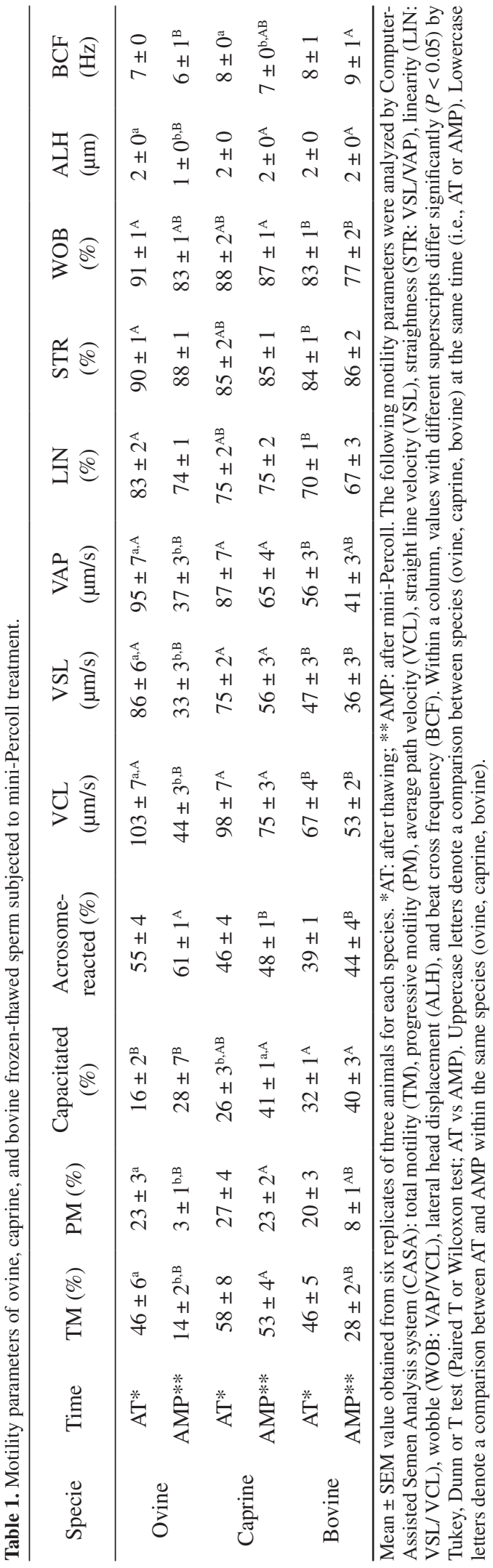


Table 2. Capacitation status of ovine, caprine, and bovine frozen-thawed sperm with or without heparin supplementation over time.

\begin{tabular}{|c|c|c|c|c|}
\hline Specie & Treatment & Time (h) & Capacitated $(\%)$ & Acrosome-reacted (\%) \\
\hline \multirow{8}{*}{ Ovine } & \multirow{4}{*}{ Control* } & 1.5 & $52 \pm 4$ & $40 \pm 4^{\mathrm{B}}$ \\
\hline & & 3.0 & $56 \pm 4^{\mathrm{A}}$ & $39 \pm 4^{\mathrm{B}}$ \\
\hline & & 6.0 & $54 \pm 8$ & $42 \pm 9$ \\
\hline & & 18.0 & $79 \pm 1^{\mathrm{A}}$ & $17 \pm 2^{\mathrm{B}}$ \\
\hline & \multirow{4}{*}{ Heparin $* *$} & 1.5 & $46 \pm 7$ & $44 \pm 7$ \\
\hline & & 3.0 & $44 \pm 8$ & $42 \pm 3^{\mathrm{B}}$ \\
\hline & & 6.0 & $66 \pm 2^{\mathrm{A}}$ & $29 \pm 3^{\mathrm{B}}$ \\
\hline & & 18.0 & $77 \pm 2$ & $20 \pm 3$ \\
\hline \multirow{8}{*}{ Caprine } & \multirow{4}{*}{ Control* } & 1.5 & $39 \pm 2$ & $53 \pm 2^{\mathrm{AB}}$ \\
\hline & & 3.0 & $38 \pm 5^{\mathrm{AB}}$ & $58 \pm 4^{\mathrm{AB}}$ \\
\hline & & 6.0 & $41 \pm 4$ & $48 \pm 6$ \\
\hline & & 18.0 & $37 \pm 4^{\mathrm{B}}$ & $59 \pm 3^{\mathrm{a}, \mathrm{A}}$ \\
\hline & \multirow{4}{*}{ Heparin** } & 1.5 & $29 \pm 4$ & $64 \pm 5$ \\
\hline & & 3.0 & $33 \pm 3$ & $64 \pm 3^{\mathrm{A}}$ \\
\hline & & 6.0 & $41 \pm 4^{\mathrm{B}}$ & $56 \pm 4^{\mathrm{A}}$ \\
\hline & & 18.0 & $41 \pm 9$ & $39 \pm 9^{b}$ \\
\hline \multirow{8}{*}{ Bovine } & \multirow{4}{*}{ Control* } & 1.5 & $38 \pm 3$ & $55 \pm 3^{\mathrm{A}}$ \\
\hline & & 3.0 & $35 \pm 4^{\mathrm{B}}$ & $60 \pm 4^{\mathrm{A}}$ \\
\hline & & 6.0 & $37 \pm 3$ & $59 \pm 4$ \\
\hline & & 18.0 & $39 \pm 3^{\mathrm{B}}$ & $56 \pm 1^{\mathrm{A}}$ \\
\hline & \multirow{4}{*}{ Heparin** } & 1.5 & $37 \pm 4$ & $58 \pm 5$ \\
\hline & & 3.0 & $36 \pm 4$ & $59 \pm 4^{\mathrm{AB}}$ \\
\hline & & 6.0 & $45 \pm 2^{\mathrm{AB}}$ & $51 \pm 3^{\mathrm{AB}}$ \\
\hline & & 18.0 & $42 \pm 3$ & $52 \pm 4$ \\
\hline
\end{tabular}

Mean \pm SEM value obtained from six replicates of three animals for each species. Within a column, values with different superscripts differ significantly $(P<0.05)$ by Dunn or T test (Paired T or Wilcoxon test; control vs heparin). Uppercase letters denote a comparison between species (ovine, caprine, bovine) at the same incubation time (1.5, 3, 6 or $18 \mathrm{~h}$ ) and treatment (control or heparin group). Lowercase letters denote a comparison between control and heparin within the same species (ovine, caprine, bovine) and incubation time $(1.5,3,6$ or $18 \mathrm{~h})$. *Control: sperm incubation in SP-TALP medium. **Heparin: sperm incubation in SP-TALP medium supplemented with $5 \mu \mathrm{g} / \mathrm{mL}$ of heparin.

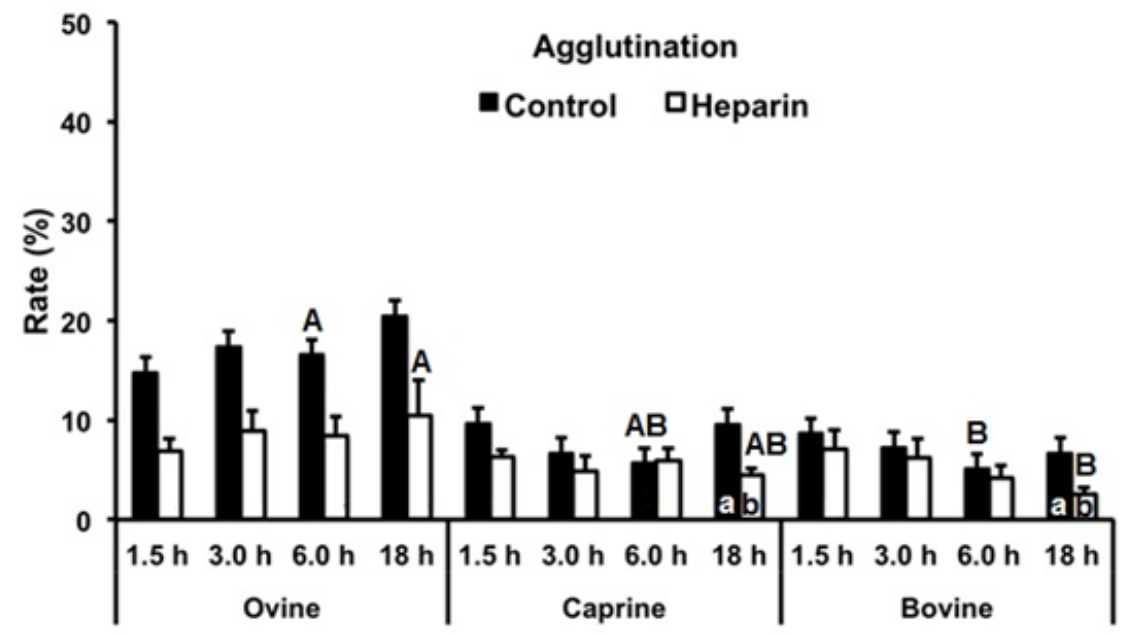

Figure 1. Rate of sperm agglutination in different domesticated ruminant species (ovine, caprine, and bovine) after incubation in medium with (Heparin) or without (Control) heparin for $18 \mathrm{~h}$ at $38.5^{\circ} \mathrm{C}$ in $5 \% \mathrm{CO}_{2}$. Data are expressed as mean \pm SEM. Different uppercase letters $(A, B)$ denote a significant difference $(P<0.05)$ between domesticated ruminant species (ovine, caprine, and bovine) within the same group (control or heparin) and same incubation time $(1.5,3,6$, or $18 \mathrm{~h})$ according to a Dunn or Wilcoxon test. Different lowercase letters $(\mathrm{a}, \mathrm{b})$ denote a significant difference $(P<0.05)$ among treatments (control or heparin) within the same incubation time $(1.5,3,6$, or $18 \mathrm{~h}$ ) and same domesticated ruminant species (ovine, caprine, or bovine) according to a paired $t$-test. Control: sperm incubation in SP-TALP medium. Heparin: sperm incubation in SP-TALP. 


\section{DISCUSSION}

Three general hypotheses were postulated: (a) cryopreserved ram sperm suffer capacitation more quickly than buck and bull sperm under the same conditions, (b) ruminant cryopreserved sperm is similarly prone to capacitation whether or not heparin is supplemented after the mini-Percoll technique, and (c) the presence of heparin in the incubation media for ruminant frozen-thawed sperm selected by mini-Percoll does not result in damage in terms of capacitation status and sperm agglutination.

The first hypothesis was not supported by the present study. Ovine, caprine and bovine frozenthawed sperm presented the same rate of increase (around 10-15\%) of capacitated cell after mini-Percoll technique. Cryopreserved samples lose phospholipids faster [1] and present a higher proportion of sperm cells with high intracellular $\mathrm{Ca}^{+2}$ [12], making the frozen-thawed sperm more susceptible to the capacitation process, independently of the species. This is in accordance with the rapid increase in the B pattern up to $1.5 \mathrm{~h}$ in all species, as demonstrated in the present study. The capacitation event that occurs in frozen-thawed sperm (i.e., cryocapacitation) is often reported in the literature. Studies using ruminant sperm reported that fresh semen needs $3 \mathrm{~h}$ of incubation to reach $50 \%$ capacitation, whereas frozenthawed semen presents higher rates of capacitated cells immediately after thawing [13]. [20] affirmed that, upon thawing, bull sperm bind to CTC in a manner very similar to freshly ejaculated sperm that have been incubated for $5 \mathrm{~h}$. The authors suggested that immediately after thawing, frozen sperm may be in an advanced capacitating state similar to fresh sperm incubated in the presence of a capacitation inducer, similar to the phenomenon observed in the current study.

Regarding the second hypothesis, the present study found no difference in capacitation status between control (absence of heparin) and heparin group in all species tested. Therefore, the second hypothesis was supported by the results of this study. The data reported in this study are similar to values found for bull, ram and buck sperm capacitation immediately after thawing [7,14,20,24]. The results indicate that ruminant frozen-thawed sperm undergo a premature capacitation, regardless of the presence of heparin, making the use of frozen-thawed semen more practi- cal and economic. The absence of heparin effect may be due to non-intact acrosome sperm (coming from capacitating event) do not react with heparin and therefore it is not possible for heparin to fix in external acrosome membrane [11].

The third hypothesis was also supported by the results of the current study. Thus, the miniPercoll technique without heparin can be a useful alternative for the preparation of frozen-thawed sperm, replacing expensive capacitation inducers. The Percoll gradient is one of the main techniques used in in vitro embryo production laboratories to separate mammal sperm from seminal plasma and/or cryoprotectant. Although we have observed reduced rates of sperm motility in all species after Percoll, its effect on the capacitation rate was also observed in all species. Percoll particles can induce the capacitation process by adhering to the sperm membranes and potentially altering them by removing some coating glycoproteins [23]. Moreover, after the selection step, this technique involves one more washing step. Our results are in accordance with the fact that the Percoll technique and repeated washing removes decapacitating components present in the PM [3] and may alter the $\mathrm{Ca}^{+2}$ distribution [19], yielding capacitated cells.

Lower rates of sperm motility were found in ovine species in the present study. These data can be supported since a species with sperm membranes are more resistant to cold-shock damage when they have a high degree of saturation of phospholipid-bound acyl moieties [21] and a high sterol to phospholipid ratio [6]. Sperm membrane cholesterol/phospholipid ratios differ among species: goat (0.59), bull (0.45), and ram (0.37) [22]. Thus, ram sperm are more sensitive to cooling and cold-shock stress than other species, and this can explain the lower motility rates found.

The precise relationship between sperm agglutination and the sperm's ability to fertilize in vivo remains unclear [28]. Sperm head-to-head agglutination during in vitro incubation could stimulate capacitation; sperm agglutination is believed to be associated with fertilizing ability $[9,10]$. In the present study, the absence of heparin led to higher agglutination rates in caprine and bovine sperm. This fact can also explain the higher capacitation rates found, and the presence of agglutination could contribute to the cell's fertilizing ability and indicate a positive feature of the sample. 


\section{CONCLUSIONS}

The present study observed similar capacitation features in domestic ruminant frozen-thawed sperm among ovine, caprine, and bovine species regardless the presence of heparin in incubation medium. Immediately after thawing followed by mini-Percoll selection and incubation for up to $18 \mathrm{~h}$, the frozenthawed sperm had a high percentage of capacitated cells, even in the absence of heparin. This suggests that ruminant frozen-thawed sperm preparation by mini-Percoll selection and incubation in media without heparin supplementation can be an efficient technique and an alternative to expensive capacitation inducers, making sperm preparation easier, cheaper, and more practical.

\section{MANUFACTURERS}

${ }^{1}$ Sigma Chemicals. St. Louis, MO, USA.
${ }^{2}$ Corning Incorporated. São Paulo, SP, Brazil

${ }^{3}$ Nikon Eclipse Ci (Nikon Corporation). Tokyo, Japan

${ }^{4}$ Microptic, Automatic Diagnostic Systems. Barcelona, Spain.

${ }^{5}$ Prism 5.01 software (GraphPad software). San Diego, CA, USA.

Funding. This work was supported by Financier of Studies and Projects (Finep) and Rio de Janeiro Research Foundation (Faperj - grant E-26/111.694/2013).

Acknowledgements. JFF and FZB are National Council for Scientific and Technological Development (CNPq) fellows. VAPA, CCSO and LRC were supported by CNPq. JMGS-F, RITPB and HFRAS were supported by Coordination for the Improvement of Higher Education Personnel (CAPES).

Ethical approval. All procedures were carried in accordance with the Animal Care Committee at the Universidade Federal Fluminense (protocol approval: 374/13).

Declaration of interest. The authors report no conflicts of interest. The authors alone are responsible for the content and writing of paper.

\section{REFERENCES}

1 Alvarez J.G. \& Storey B.T. 1992. Evidence for increased lipid peroxidation damage and loss of superoxide dismutase activity as a mode of sublethal cryodamage to human sperm during cryopreservation. Journal of Andrology. 13: 232241. https://doi.org/10.1002/j.1939-4640.1992.tb00306.x

2 Bedford J.M. 1983. Significance of the need for sperm capacitation before fertilization in eutherian mammals. Biology of Reproduction. 28: 108-120. https://doi.org/10.1095/biolreprod28.1.108.

3 Cesari A., Kaiser G.G. \& Mucci N. 2006. Integrated morphophysiological assessment of two methods for sperm selection in bovine embryos production in vitro. Theriogenology. 66: 1185-1193. https://doi.org/10.1016/j.theriogenology.2006.03.029

4 Chamberland A., Fournier I.V., Tardff S., Sirard I.M.A., Sulhvan I.R. \& Badey J.L. 2001. The effect of heparin on motility parameters and protein phosphorylation during bovine sperm capacitation. Theriogenology. 55: 823-835. https://doi.org/10.1016/S0093-691X(01)00446-0

5 Cormier N., Sirard M.A. \& Bailey J.L. 1997. Premature capacitation of bovine spermatozoa is initiated by cryopreservation. Journal of Andrology. 18: 461-468. https://doi.org/10.1002/j.1939-4640.1997.tb01953.x

6 Darin-Bennett A. \& White I.G. 1977. Influence of the cholesterol content of mammalian spermatozoa on susceptibility to cold-shock. Cryobiology. 14: 466-470. https://doi.org/10.1016/00112240(77)90008-6

7 García-Álvarez O., Maroto-Morales A., Jiménez-Rabadán P., Ramón M., Del Olmo E., Iniesta-Cuerda M., AnelLópez L., Fernández-Santos M.R., Garde J.J. \& Soler A.J. 2015. Effect of different media additives on capacitation of frozen-thawed ram spermatozoa as a potential replacement for estrous sheep serum. Theriogenology. 84: 948-955. https://doi.org/10.1016/j.theriogenology.2015.05.032.

8 Hashimoto S., Minami N., Takakura R., Yamada M., Imai H. \& Kashima N. 2000. Low oxygen tension during in vitro maturation is beneficial for supporting the subsequent development of bovine cumulus-oocyte complexes. Molecular Reproduction and Development. 57: 353-360. https://doi.org/10.1002/1098-2795(200012)57:4<353::AID-MRD7>3.0.CO;2-R

9 Harayama H., Miyake M., Shidara O., Iwamoto E. \& Kato S. 1998. Effects of calcium and bicarbonate on headto-head agglutination in ejaculated boar spermatozoa. Reproduction Fertility and Development. 10: 445-450. https:// doi.org/10.1071/RD98124

10 Harrison R.A. 1997. Sperm plasma membrane characteristics and boar semen fertility. Journal of Reproduction Fertility. 52(Suppl): 195-211.

11 Januskauskas A., Gil J., Soderquist L. \& Rodriguez-Martínez H. 2000. Relationship between sperm respond to glycosaminoglycans in vitro and non-return rates of Swedish dairy AI bulls. Reproduction in Domestic Animals. 35: 207-212. https://doi.org/10.1046/j.1439-0531.2000.00212.x 
12 Kadirvel G., Kumar S., Kumaresan A. \& Kathiravan P. 2009. Capacitation status of fresh and frozen-thawed buffalo spermatozoa in relation to cholesterol level, membrane fluidity and intracellular calcium. Animal Reproduction Science. 116: 244-253. https://doi.org/10.1016/j.anireprosci.2009.02.003

13 Kadirvel G., Kathiravan P. \& Kumar S. 2011. Protein tyrosine phosphorylation and zona binding ability of in vitro capacitated and cryopreserved buffalo spermatozoa. Theriogenology. 75: 1630-1639. https://doi.org/10.1016/j.theriogenology.2011.01.003

14 Miah A.G., Salma U., Sinha P.B., Holker M., Tesfaye D., Cinar M.U., Tsujii H., Schellander K. 2011. Intracellular signaling cascades induced by relaxin in the stimulation of capacitation and acrosome reaction in fresh and frozen-thawed bovine spermatozoa. Animal Reprodution Science. 125: 30-41. https://doi.org/10.1016/j.anireprosci.2011.03.010

15 Olivares C.C.S., Fonseca J.F., Camargo L.S.A., Souza-Fabjan J.M.G., Rodrigues A.L.R. \& Brandão F.Z. 2015. Comparison of different methods of goat sperm selection and capacitation for optimization of assisted reproductive technologies. Small Ruminant Research. 127: 44-49. https://doi.org/10.1016/j.smallrumres.2015.04.009

16 Olivares C.C.S., Souza-Fabjan J.M.G., Fonseca J.F., Balaro M.F.A., Freitas V.J.F., Oliveira R.V. \& Brandão F.Z. 2017. Comparison of Different Sperm Selection Techniques in Ram Frozen-Thawed Sperm. Acta Scientiae Veterinariae. 45: 1431-1437.

17 Parrish J.J., Susko-Parrish J., Winer M.A. \& First N.L. 1988. Capacitation of bovine sperm by heparin. Biology of Reproduction. 38(5): 1171-1180. https://doi.org/10.1095/biolreprod38.5.1171

18 Parrish J.J. 2014. Bovine in vitro fertilization: in vitro oocyte maturation and sperm capacitation with heparin. Theriogenology. 81: 67-73. https://doi.org/10.1016/j.theriogenology.2013.08.005

19 Pérez L.J., Valcarcel A., De Las Heras M.A., Moses D.F. \& Baldassarre H. 1995. In vitro capacitation and induction of acrosomal exocytosis in ram spermatozoa as assessed by the chlortetracycline assay. Theriogenology. 45: 1037-1046. https://doi.org/10.1016/0093-691X(96)00031-3

20 Pons-Rejraji H., Bailey J.L. \& Leclerc P. 2009. Cryopreservation affects bovine sperm intracelular parameters associated with capacitation and acrosome exocytosis. Reproduction Fertility and Development. 21: 525-537. https://doi. org/10.1071/RD07170

21 Poulos A., Darin-Bennett A. \& White I.G. 1973. The phospholipid-bound fatty acids and aldehydes of mammalian spermatozoa. Comparative Biochemistry and Physiology Part B: Comparative Biochemistry. 46: 541-549. https://doi. org/10.1016/0305-0491(73)90094-1

22 Rana A.P., Majumder G.C., Misra S. \& Ghosh A. 1991. Lipid changes of goat sperm plasma membrane during epididymal maturation. Biochim Biophys Acta. 1061 (2): 185-196. https://doi.org/10.1016/0005-2736(91)90284-f

23 Rosenkranz C. \& Holzmann A. 1997. The effect of sperm preparation on the timing of penetration in bovine in vitro fertilization. Animal Reproduction Science. 46: 47-53. https://doi.org/10.1016/S0378-4320(96)01606-5

24 Salmon V.M., Leclerc P. \& Bailey J.L. 2016. Cholesterol-loaded cyclodextrin increases the cholesterol content of goat sperm to improve cold and osmotic resistance and maintain sperm function after cryopreservation. Biology of Reproduction. 94(4): 1-12. https://doi.org/10.1095/biolreprod.115.128553.

25 Souza J.M.G., Duffard N., Bertoldo M.J., Locatelli Y., Corbin E., Fatet A., Freitas V.J. \& Mermillod P. 2013. Influence of heparin or the presence of cumulus cells during fertilization on the in vitro production of goat embryos. Animal Reproduction Science. 138: 82-89. https://doi.org/10.1016/j.anireprosci.2013.02.012

26 Sreenivas D., Thomas V.M., Kaladhar D.S.V.G.K., Yarla N.S., Palni Samy A., Varahalarao V., Preethi R. 2013. In vitro production of sheep embryos in CR1aa medium supplemented with L-ascorbic acid: effects on morula and blastocyst development. Journal of Tissue \& Engineering. 5: 87-193. https://doi.org/10.4172/1948-593X.1000097

27 Tonieto R.A., Goularte K.L., Gastal G.D.A., Schiavon R.S., Deschamps J.C. \& Lucia Jr. T. 2010. Cryoprotectant effects of trehalose and low-density lipoprotein in extenders for frozen ram semen. Small Ruminant Research. 93: 206-209. https://doi.org/10.1016/j.smallrumres.2010.05.003

28 Yang D.H., McMillan A.G., Standley N.T., Shannon P. \& Xu Z.Z. 2012. Extracellular calcium is involved in egg yolk-induced head-to-head agglutination of bull sperm. Theriogenology. 78: 1476-1486. https://doi.org/10.1016/j. theriogenology.2012.06.021 